\title{
A Novel ASNO Segmentation Technique for Segmenting the JUXTA Vascular Region
}

\author{
Arun B. Mathews ${ }^{1 *}$, M.K. Jeyakumar ${ }^{2}$ \\ ${ }^{1}$ Research Scholar, Department of Computer Science, Noorul Islam Centre for Higher Education, Tamil Nadu, India. \\ ${ }^{2}$ Professor, Department of Computer Applications, Noorul Islam Centre for Higher Education, Kumaracoil, Tamil Nadu, India. \\ E-mail:jeyakumarmk@yahoo.com \\ *Corresponding author E-mail:marthomat@gmail.com
}

\begin{abstract}
In this modern era the clinical laboratory has greater attention to produce an accurate result for every test particularly in the area of lung tumour. The lung tumour is very essential to detect as well as to follow the treatment of many diseases like benign, malignant etc. This paper is focusing on the segmentation part to find the juxta vascular region. For finding the juxta vascular region in lung three stages are used. First stage is the image acquisition here input lung image is read and then resized. Second stage is the image pre-processing here improved linear iterative clustering technique is used.Third stage is the segmentation here the adjustable surface normal overlap is used. While using the above stages the output for juxta vascular region in the segmentation part is segmented clearly.

The juxta vascular region is not clearly found in the previous paper. The research gap for this paper is to find the juxta vascular region in the lung. This juxta vascular region is present in the right side of the lung. Using the Adjustable Surface Normal Overlap (ASNO) segmentation the juxta vascular region is segmented clearly.
\end{abstract}

Keywords: Lung tumour, improved linear iterative clustering, adjustable surface normal overlap, JUXTA vascular region.

\section{Introduction}

A small plug on the lung is identified as pulmonary nodules. Lung nodes is an unimportant masses of tissue in the lung and are quite common. A pulmonary nodules look like round, white glooms on the Computerized Tomography (CT) scan. To detect whether it is tumorous or not further scans are used CT scan, Positron Emission Tomography(PET) scan, Bronchoscopy or tissue biopsy etc. By using the CT scan we get a clear image for nodule and size, shape and location for nodules. If the nodule is lesser and flat then the doctor will check the monitor whether the size and shapes are changing or not, if it is changing then repeat the CT scan for sometimes at fixed intervals. The Lung cancer is the second most commonly diagnosed cancer and the lung is a most frequent site of metastasis from other cancers that manifest as pulmonary nodules. The Chest computed tomography (CT) is the most sensitive diagnostic imaging modality for the detection of lung cancer and the resolution of any equivocal abnormalities detected on chest radiographs. Recently, CT techniques have been applied to screening for the detection of the lung cancer in high-risk populations and have been shown to be promising for detection of early lung cancers. Lung nodules are generally nearby 5 millimetres to 30 millimetres in size. And a bigger lung nodule, is 30 millimetres in size, this is said to be malignant. The hazard for cancer upsurges when: a nodule is bulky, the node look like a pointed surface, a chain-smoker, agene of lung tumour, a history of chronic obstructive lung disease, over age 60.

\section{Related Work}

JaspinderKaur et.al used various Computer Aided Diagnosis (CAD) systems for the early diagnose of lung tumour [1]. J. M. Kuhnigk et.al have some limits and problems to segment and classify the lung nodule in these systems which include[2]1) pulmonary vessel segmentation and voxel classification is fully needed, which is repeatedly a difficult process and sensitive to the certain threshold and X. Zhou et.al 2) inaccuracy in relating Hough transform to identify curved surfaces [3] and also S. Saita et.al 3) inability of detecting abnormal lung arrangements [4].

Omnia Elsayed et.al proposed three step to detect the lung nodule the first step is the pre-processing here changing the pixel values into Hounsfield units and then relating a region growing method. Second step starts with thresholding and the lung image is segmented by Hessian method for vascular tree segmentation. And the third step is the classification here using these linear classifier, quadratic classifier, Parzen classifier, and Neural Network classifier the lung nodules is classified [5].[6] In the initial stage the lung cancer is very hard to classify by Smritikushwaha and P.Maya using the fuzzy C-means clustering analysis the lung nodule is segmented after that using DWT and MWT transforms the feature is extracted and by SVM classification the four kinds of lung nodules, i.e., wellcircumscribed, vascular-ized, juxta-pleural and pleural-tail is classified. And by using LBP the cancer cell is detected.

A review paper of Rotem Golan et.al proposed a backpropagation algorithm to train the Convolutional Neural Network for differentiating the size and shape of the lung nodule and also to extract volumetric feature from the input lung image [7].A. 
Mangalakshmi et.al proposed a contextual analysis to merger the lung nodule and adjacent anatomical structures. There are three main stages in this system (i) To build concentric multilevel partition an adaptive patch-based division is used (ii) To combine intensity, texture, and gradient information a new feature set is planned for feature description and (iii) And the classifier used here is the contextual latent semantic based classifier [8].

Tao Zhou et.al presented a paper to classify the lung nodules. Firstly, CT image is analysed using 42 dimensional feature. Secondly, based on feature level fusion these features are condensed for five eras with roughest. Thirdly, using SVM classification a grid optimization model optimized to classify the pulmonary nodules [9].

I. Mary SajinSanju and Sonali Singh proposed a different method of classification, segmentation and detection techniques to find the lung nodules. Initially the pre-processing technique to remove the Gaussian noise the Gaussian filter is used. Secondly, Otsu thresholding is applied to segment the Region of Interest (ROI). Lastly, the Support Vector Machine (SVM) is applied which will classify the extracted feature vectors. Based on the second order grey level co-occurrence matrix features the SVM will classify the images into normal or abnormal [10].A review paper RabiaNaseem et.al proposed an automated method to classify the lung nodules. Using the false positive reduction the lung nodule is classified as normal and abnormal region [11].

Qaisar Abbas proposed a deep learning algorithm to classify the lung nodule. Without doing pre or post-processing steps a Multilayer combination of the Convolutional Neural Network (CNN), Recurrent Neural Networks (RNNs) and soft max linear classifiers are integrated to develop the Lung-Deep. Compared to the use of manual segmentation in this study done by a professional radiologist. Hence, the development of this CAD tool for pattern classification is having a great clinical importance and it assists radiologists to better identify the lung-related disease [12].YenyYim et.al proposed three steps to extract the lung nodule. Firstly, by an inverse seeded region growing the lungs and airways are extracted. Secondly, using three-dimensional region growing the trachea and large airways are outlined from the lungs. Thirdly, by subtracting the first two steps accurate lung area borders are obtained [13].

The earlier CAD systems [14]-[21],[26]-[28] based on three steps, i.e. Segmentation, feature extraction, the collection of most projecting features and the last step is to classify these discriminative features for recognition of lung disease. A sum of supervised FP reduction techniques have been reported for the characterization of INCs, such as Linear Discriminant Analysis (LDA) [22], [23], artificial neural network (ANN) [24], [21]-[23]. Study by Jiang and colleagues had systematically examined and comparatively analysed the alteration of DNA methylation at genome and gene levels in Xuanwei lung cancer tissues, as well as BaP-treated cells and mouse samples [25].

This paper is mainly focusing only on the pre-processing and segmentation step.

The main contribution of this paper is to follow the below techniques:

i) The existing pre-processing technique Gaussian filter here the noises are not clearly remove so far another technique improved linear iterative clustering is used.

ii) Improved Linear Iterative Clustering is a new technique to improve the quality of the image and to enhance the image.

iii) Some segmentation technique is depending only on the number of clusters, region of interest etc. (i.e. Otsu thresholding) so new technique is used i.e. Adjustable Surface Normal Overlap.

iv) Adjustable Surface Normal Overlap is the new technique here each lesion is viewed clearly to find the juxta vascular region.

The organization of the paper is as follows: Section I emphasizes introduction and the studies of several researches papers, section
II explains the methodology, section IV states the obtained simulation results and their discussion and section $\mathrm{V}$ concludes the paper.

\section{Steps to Segment the JUXTA Vascular Region}

The aim of the futuretechnique is to categorise the regular and irregular cell in lung nodules. It mainly consists of five stages: Image acquisition, image pre-processing, segmentation, feature extraction and classification. The first stage is the image acquisition here the input image is taken from the LIDC (Lung Image Database Consortium) database. The second stage is the image pre-processing here Improved Linear Iterative Clustering (ILIC) method is used to section the lung region. The third stage is the segmentation here the lung tumour is segmented using the Adjustable Surface Normal Overlap (ASNO). The fourth stage is the feature extraction here two features are used: GLCM and FOS .And the fifth stage is the classification here the lung nodule is classified using novel GWO based SVM.

\section{Block Diagram}

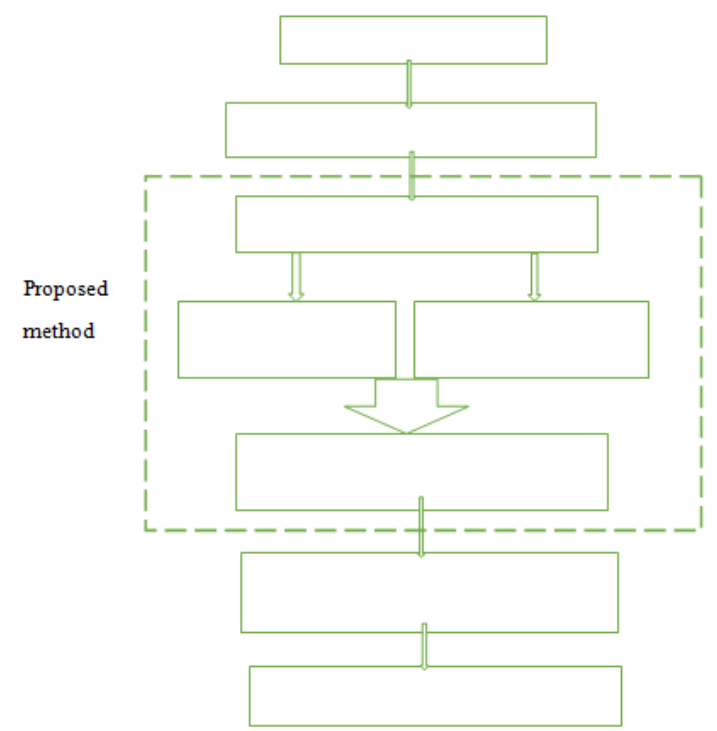

Figure 1: Block diagram for segment the JUXTA vascular region

This paper is focusing on pre-processing and segmentation which is highlighted in the figure 1 .

\section{Image Acquisition}

Image acquisition is the process of retrieving image from an imaging modality. The main work of image acquisition is to read and resize the input lung image. The input lung image is taken from the LIDC (Lung Image Database Consortium)Computerized Tomography CT scan images are better able to show contrast difference among nodules and adjacent organs.

\section{Image Pre-Processing}

The main purpose of the image pre-processing is not simply the elimination of the unwanted noise and background information but also the alteration of the image pixel values. The histogram equalization techniques used for enhancing the images are of two types via frequency domain and spatial domain. It considerably improves the perception and interpretability of the image boundaries for human viewers. One of the frequency domain techniques is histogram equalization and it is used to improve the contrast of the images. 
Histogram equalization is a method to process images in order to adjust the contrast of an image by modifying the intensity distribution of the histogram. The objective of this technique is to give a linear trend to the cumulative probability function associated to the image.

In the Improved Linear Iteration Clustering (ILIC) algorithm, the super pixels are equally sized. To section the lung area super pixel is applied. While using the Improved Linear Iterative Clustering algorithm cluster boundaries of the pixels among the super-pixels is considered by an integer 0 and the additional information of the pixels indicate that it energies to the particular super-pixels. For every pixel, it may possibly be collected by their neighbours which have the related grey values. The parallel connection of the super-pixels is calculated, and the result is formed as a parallel matrix. At last it is done based on the colour such as black, white and grey.

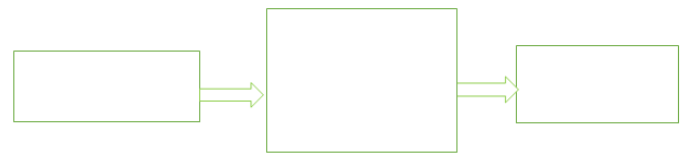

Figure 2: Block diagram of image pre-processing

Figure 2 shows the block diagram pre-processed image of the lung tumour using Improved Linear Iterative Clustering.

\section{Segmentation}

In segmentation the nodule is segmented using the Adjustable Surface Normal Overlap. Initially detection of lung tumours (observable on chest radiographs as nodules) may increase the patients, but the nodule detection problem is a complicated task. It is somewhat low-contrast with white circular objects in the lung region. To differentiate true nodules from shadows, vessels, and ribs there is a trouble in the CAD system.

The Adjustable Surface Normal Overlap (ASNO) is critical for segmenting the lung nodules. Each layer is accumulated a score related to the number of surface normal that pass through or near it. Generally lung nodules tend to have some convex regions on their surfaces and thus, the inward pointing surface normal vectors, near these features tend to intersect or nearly intersect within the lung.

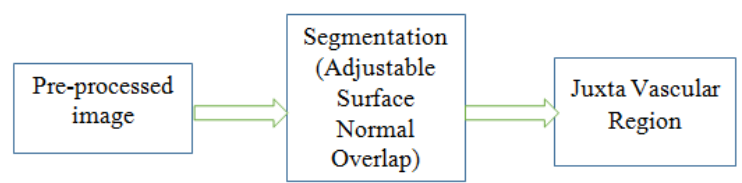

Figure 3: Block diagram of segmentation

Figure 3 shows the block diagram of segmentation using Adjustable Surface Normal Overlap to find the Juxta Vascular Region.

\section{Feature Extraction}

\section{i) FOS Features}

- FOS feature is a Histogram based features

- The histogram-based features used here is the first order statistics.

- It consists of Mean, Variance, Skewness and Kurtosis.

- Let $\mathrm{z}$ be a random variable indicating the image grey levels and $\mathrm{p}(\mathrm{zi}), \mathrm{i}=0,1,2,3, \ldots \ldots . \mathrm{L}-1$, be the resultant histogram.

- Where $\mathrm{L}$ is the number of different gray levels.

- Using the above mentioned histogram the features are calculated.

\section{Mean}

The mean contributes the normal grey level of all area and it is useful only as anunevenhint of intensity not really texture.

Mean is shown in equation 1

Mean $\mu_{i}=\frac{1}{N} \sum_{j-1}^{N} f_{i j}$

\section{Standard Deviation}

Standard deviation is used for depicting the gap of the enhanced image. Standard deviation is shown in equation 2

$\mathrm{SD}=\sigma_{\mathrm{i}}=\left(\frac{1}{\mathrm{~N}} \sum_{\mathrm{j}-1}^{\mathrm{N}}\left(\mathrm{f}_{\mathrm{ij}}-\mu_{\mathrm{i}}\right)^{2}\right)^{\frac{1}{2}}$

\section{Skewness}

An amount of irregularity of the grey levels about the sample mean is known as Skewness as shown in equation 3

$$
S_{k}(X)=\left(\frac{1}{m \times n}\right) \frac{\Sigma\left(f(x, y)-M^{3}\right.}{S D^{3}}
$$

\section{(d)Kurtosis}

Kurtosis is a measure of how outlier-prone a distribution is. It describes the contour of the tail of the histogram. For the random variable $X$, the Kurtosis is denoted as $K_{u r t}(X)$ and it is shown in equation 4

$K_{u r t}(X)=\left(\frac{1}{m \times n}\right) \frac{\Sigma\left(f(x, y)-M^{4}\right.}{S D^{4}}$

\section{Entropy}

The measure of uncertainty in the image grey levels is recognised as entropy. The entropy is considered to describe the randomness of the textural image and is shown in equation 5

E

$=-\sum_{x=0}^{m-1} \sum_{y=0}^{n-1} f(x, y) \log _{2} f(x, y)$

Where

$\mathrm{N}$ is the total number of pixels in the image.

\section{(ii) GLCM}

GLCM stands for Grey Level Co-occurrence Matrix. GLCM is a fixed statistical device for removing second order texture information from the lung image. The sum of rows and columns is equivalent to the number of individual grey levels in the GLCM matrix.

\section{Energy}

The sum of squared GLCM elements is known as energy. The other name of energy is regularity. The method pixel pair duplication is also known as as textural uniformity. It detects disarrays in textures. And it is shown in equation 6

$E n=\sqrt{\sum_{x=0}^{m-1} \sum_{y=0}^{n-1} f^{2}(x, y)}$ 


\section{Contrast}

An amount of intensity of a pixel is known as Contrast. And its neighbour is over the image, which is shown in equation 7

$C_{o n}=\sum_{x=0}^{m-1} \sum_{y=0}^{n-1}(x-y)^{2} f(x, y)$

\section{Correlation}

The spatial dependencies between the pixels are described in the Correlation feature and it is shown in equation 8

$$
C_{o r r}=\frac{\sum_{x=0}^{m-1} \sum_{y=0}^{n-1}(x, y) f(x, y)-M_{x} M_{y}}{\sigma_{x} \sigma_{y}}
$$

\section{Inverse Difference Moment (IDM) or Homogeneity:}

A measure of incomplete homogeneity of an image is known as Inverse Difference Moment. And is shown in equation 9

$$
I D M=\sum_{x=0}^{m-1} \sum_{y=0}^{n-1} \frac{1}{1+(x-y)^{2}} f(x, y)
$$

\section{Novel GWO Based SVM}

SVM stands for Support Vector Machine. Support vector machines is a supervised learning representations for analysing data and recognizing patterns, used for classification. The GWO algorithm is deployed to choose near optimal combinations and a set of approximately optimal weights for the training set of images. Moreover, it is active to optimize the SVM parameter in the classification process.

GWO-SVM is an optimized based classification works on the hunting strategy of grey wolves and their leadership quality. According to leadership and hunting strategy, the grey wolves are divided into four divisions such as alpha, beta, delta and omega. The algorithm is implemented based on hunting, searching for prey, encircling prey, and attacking prey of wolf behaviour. The SVM is optimized by the GWO algorithm. The exploration and exploitation allows the classifier to share the information through the search space with sudden jump in promising area. The locally optimal solution is avoided by using several candidate solutions. The circle shaped solution searching is obtained for higher dimensions of search space.The algorithm gives the solved and optimized parameters and provides a clear optimized value for the SVM classifier.

\section{Result and Discussion}

In this section the result and discussion is focusing only on the image pre-processing and segmentation techniques. The input lung image is taken from Lung Image Database Consortium (LIDC) database, to segment the juxta vascular region .The input lung image is pre-processed by using clustering methods as improved linear iterative clustering. To segment the lung an adjustable surface normal overlap on the CAD system is used.

\section{A. Software Description}

The result is simulated by using MATLAB 2016a software. MATLAB code is inserted with statistical and image processing tools. The input lung image of 40 patients obtained from LIDC database are analysed and examined to obtain the segmentation results of the proposed system.

\section{B. Experimental Result}

The following stages shows the result of the input lung image from the LIDC database. They are: image acquisition, image preprocessing and segmentation.

- First stage is the image acquisition here the input image is read and then resized.

- Second stage is the image pre-processing here the improved linear iterative clustering technique is done and it is based on color.

- Third stage is the adjustable surface normal overlap here each and every lesion is segmented to find the juxta vascular region.

\section{Stage 1: Image Acquisition}

In image acquisition the input lung image is taken from the LIDC database. After taking the database the image is read and then resized.

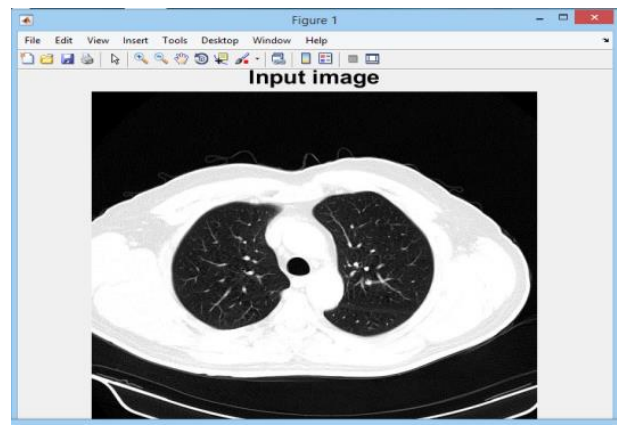

Figure 4: Input lung image from the LIDC database

Figure 4shows the input lung image from the Lung Image Database Consortium (LIDC) database and it is read and then resized.

\section{Stage 2: Image Pre-processing}

The pre-processing technique is the improved linear iterative clustering technique. Here the input is given to a pre-processing technique their histogram is done.After that the histogram is given to the histogram equalization of the input image it is based on the intensity value. Next the histogram equalization output is given to the improved linear iterative clustering technique their clustering is based on the color i.e. white, black and grey. While using this technique the image is enhanced and improved the quality of the image.

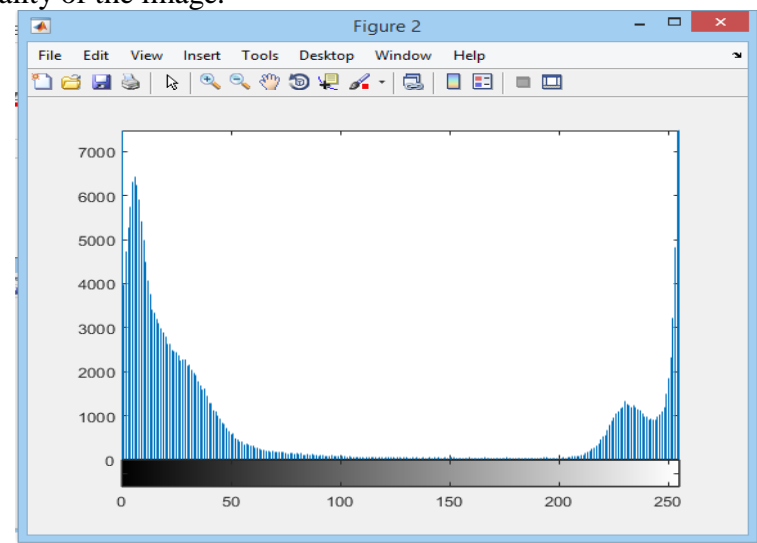

Figure 5: Histogram of the input image 


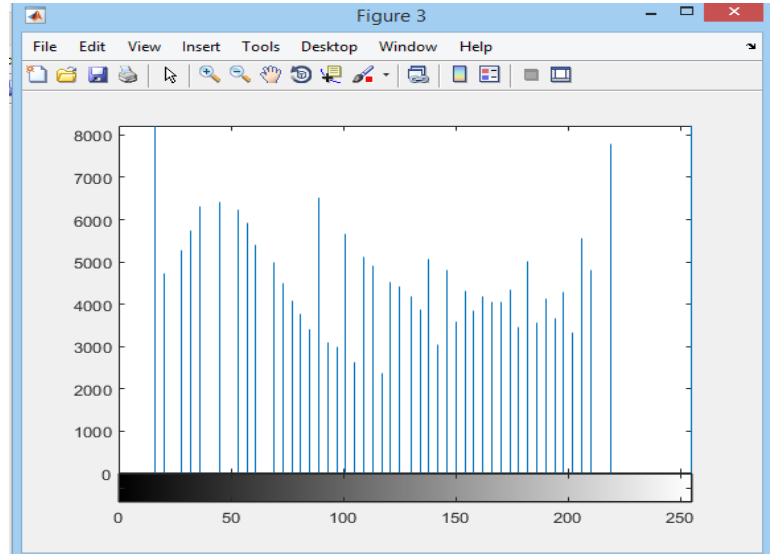

Figure 6: Histogram equalization of the input image based on the intensity value

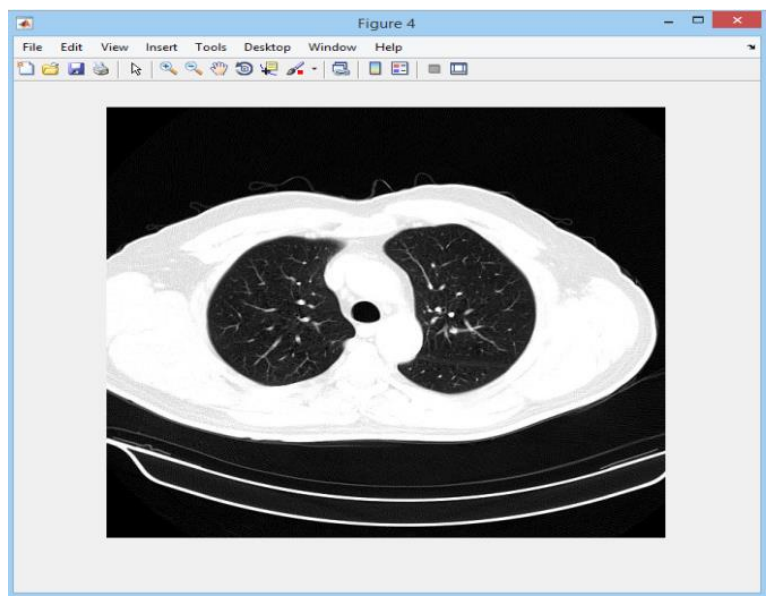

Figure 7: Histogram equalization of the image

Figure 5, 6 and 7 shows the output of the histogram equalization of the input lung image. Here the figure 6 gives the histogram equalization of the input image which is based on the intensity value.

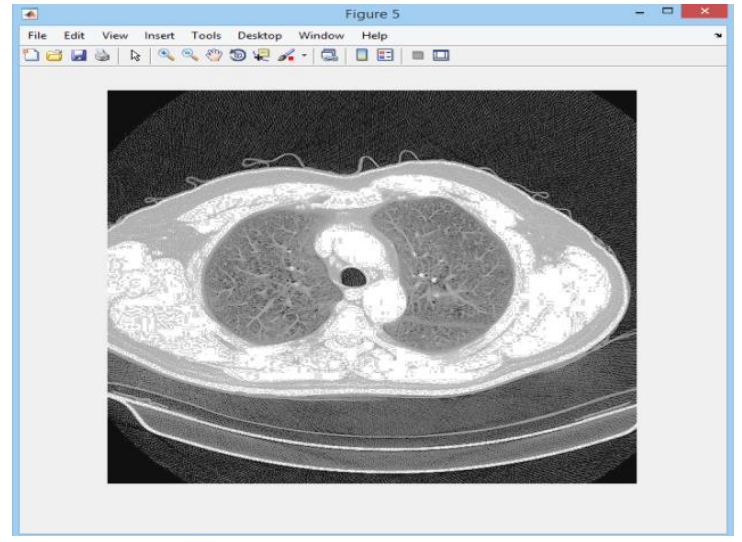

Figure 8: Output of improved linear iterative clustering

Figure 8 shows the output of the improved linear iterative clustering technique here the image is enhanced and the quality of the image is improved based on the color i.e. White, black and grey.

\section{Stage 3: Segmentation}

After image pre-processing the pre-processed image is given to the segmentation technique (adjustable surface normal overlap) to segment the tumour. While using these technique each and every lesion is viewed clearly.

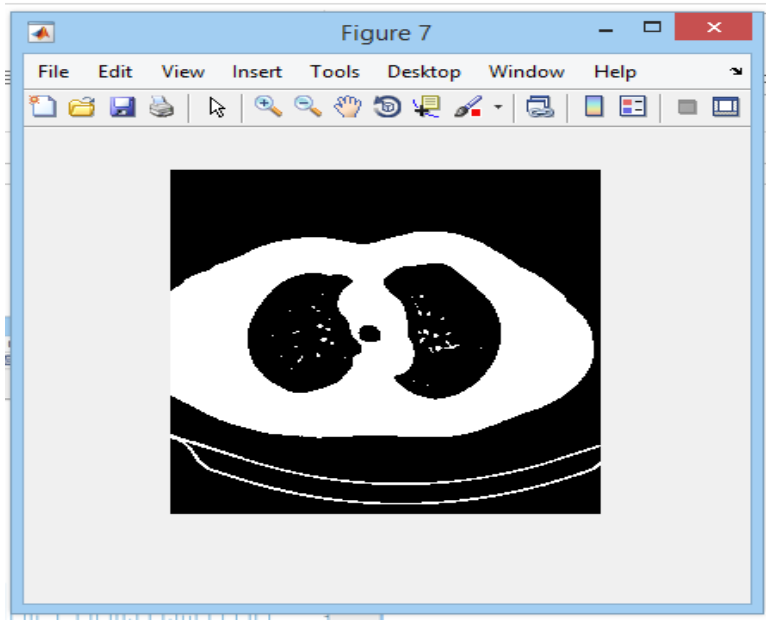

Figure 9: Adjustable surface normal overlap

Figure 9 shows that the each lesion is viewed based on the adjustable surface normal overlap. For segmenting the juxta vascular region we do the adjustable surface normal overlap technique.

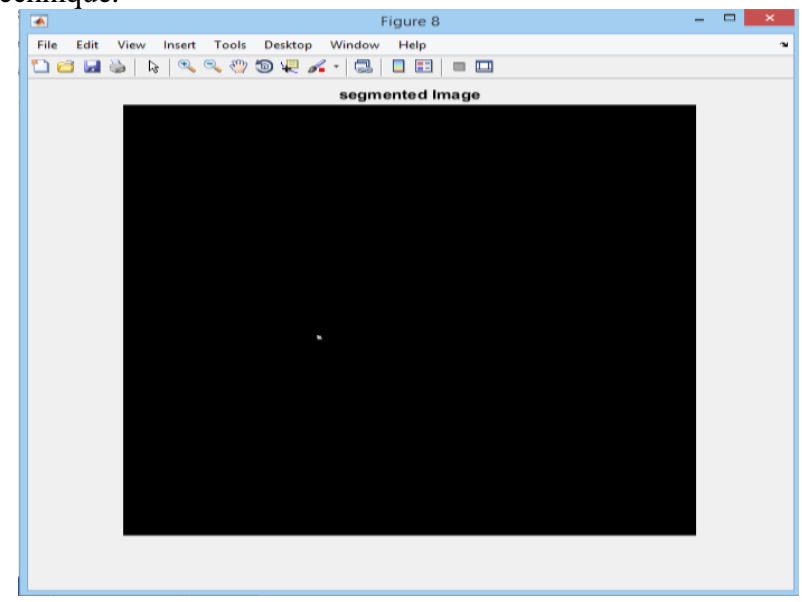

Figure 10: Output of the JUXTA vascular region

Figure 10 shows output of juxta vascular regionusing the Adjustable Surface Normal Overlapeach and every lesion is segmented. The juxta vascular region based on the segmentation technique. Adjustable surface normal overlap technique is identifying the tumour cell and segment the cell.[33]

\section{Conclusion}

In this paper the input lung image is taken from the Lung Image Database Consortium (LIDC) database. At first the input lung image is given to the image acquisition their lung image is read and then resized. The resized image is then given to preprocessing technique. The pre-processing is done by histogram equalization technique and improved linear iterative clustering technique their image is enhanced and it improves the quality of the image. The pre-processed image is given to the segmentation technique as Adjustable surface normal overlap. Using the Adjustable surface normal overlap technique the juxta vascular region is segmented. We conclude that our segmentation technique gives the high accuracy to the classification.

\section{References}

[1] Kaur J, Garg N \& Kaur D, “A survey of lung cancer detection techniques on CT scan images", International Journal of Scientific \& Engineering Research, Vol.5, No.6, (2014).

[2] Kuhnigk JM, Hahn H, Hindennach M, Dicken V, Krass S \& Peitgen HO, "Pulmonary lobe segmentation by anatomy-guided 3- 
DWatershed transform”, Proc. SPIE Med. Imag., Vol.5032, (2003), pp.1482-1490.

[3] Zhou X, Hayashi T, Hara T, Fujita H, Yokoyama R, Kiryu T \& Hoshi H, "Automatic recognition of pulmonary lobes and fissures frommulti-slice CT images", Proc. SPIE Med. Imag., Vol.5370, (2004), pp.1629-1633.

[4] Saita S, Kubo M, Kawata Y, Niki N, Ohmatsu H \& Moriyama N, "An algorithm for the extraction of pulmonary fissures from lowdosemultislice CT image", Syst. Comput. Japan, Vol.37, (2006), pp.63-76.

[5] Omnia E, Khaled M, Mohamed K \& Hatem AK, "Automatic Detection of the Pulmonary Nodules from CT Images", SAI Intelligent Systems Conference, (2015).

[6] Smritikushwaha \& Maya P, "Analysis of Lung Nodule and Classification based on SVM Classifier and Fuzzy C-Means Clustering for Effective Segmentation", International Journal of Digital Application \& Contemporary, Vol.4, No.8, (2016).

[7] Golan R, Jacob C \& Denzinger J, "Lung nodule detection in CT images using deep convolutional neural networks", IEEE International Joint Conference on Neural Networks (IJCNN), (2016), pp.243-250.

[8] Mangalakshmi A, Saranya G, Sobana Devi I \& Syamala Devi V, "Lung Nodule Classification Using Deep Features in Ct Images", International Journal of Advanced Research in Computer Science and Software Engineering, Vol.6, No.3, (2016).

[9] Tao Z, Huiling L, Junjie Z \& Hongbin S, "Pulmonary Nodule Detection Model Based on SVM and CT Image Feature-Level Fusion with Rough Sets", Hindawi Publishing Corporation BioMed Research International, (2016), pp.1-13.

[10] Sonali S \& Mary SajinSanju I, "The detection of pulmonary nodules in CT images using heuristic approach segmentation and classification", ARPN Journal of Engineering and Applied Sciences, Vol.11, No.9, (2016), pp.5916 5920.

[11] Naseem R, Alimgeer KS \& Bashir T, "Recent trends in Computer Aided diagnosis of lung nodules in thorax CT scans", IEEE International Conference on Innovations in Electrical Engineering and Computational Technologies (ICIEECT), (2017), pp.1-12.

[12] Qaisar A, "Lung-Deep: A Computerized Tool for Detection of Lung Nodule Patterns using Deep Learning Algorithms", International Journal of Advanced Computer Science and Applications, Vol.8, No.10, (2017), pp.112-116.

[13] Yim Y, Hong H, \& Shin YG, "Hybrid lung segmentation in chest CT images for computer-aided diagnosis", Proceedings of 7 th International Workshop on Enterprise networking and Computing in Healthcare Industry, (2005), pp.378-383,

[14] Pastorino U, "Early detection of lung cancer", Respiration, Vol.73, No.1, (2006), pp.5-13.

[15] Guy JA \& Lehmann HP, "After detection: the improved accuracy of lung cancer assessment using radiologic computer-aided diagnosis", Academic Radiology, Vol.23, (2016), pp.186-191.

[16] Chan HP, Hadjiiski L, Zhou C \& Sahiner B, "Computer-aided diagnosis of lung cancer and pulmonary embolism in computed tomography A review", Academic Radiology, Vol.15, (2008), pp.535-555.

[17] Theresa MM \& Bharathi VS, "Computer aided diagnostic (CAD) for feature extraction of lungs in chest radiograph using different transform feature", Biomedical Research, (2017), pp.1-8.

[18] Way TW, Sahiner B, Chan HP, Hadjiiski L, Cascade PN \& Chughtai A, "Computer-aided diagnosis of pulmonary nodules on CT scans: improvement of classification performance with nodule surface features", Medical Physics, Vol.36, (2009), pp.3086-3098.

[19] Messay T, Hardie RC \& Rogers SK, "A new computationally efficient CAD system for pulmonary nodule detection in CT imagery", Medical Image Analysis, Vol.14, (2010), pp.390-406.

[20] Reeves A.P., Chan A.B., Yankelevitz D.F., Henschke C.I., Kressler B., Kostis W.J, "On measuring the change in size of pulmonary nodules", IEEE transactions on medical imaging, vol.25, pp.435-450, 2006.

[21] De CarvalhoFilho AO, Silva AC, De Paiva AC, Nunes RA \& Gattass M, "Computer-aided diagnosis system for lung nodules based on computed tomography using shape analysis, a genetic algorithm and SVM", Medical \& biological engineering \& computing, (2016), pp.1-8.

[22] Messay T, Hardie RC \& Rogers SK, "A new computationally efficient CAD system for pulmonary nodule detection in CT imagery", Med. Image Anal., Vol.14, No.3, (2010), pp.390-406.

[23] Lee Y, Hara T, Fujita H, Itoh S \& Ishigaki T, "Automated detection of pulmonary nodules in helical CT images based on an improved template matching technique”, IEEETrans.Med.Imag., Vol.20, No.7, (2001), pp.595-604.

[24] Awai K, Murao K, Ozawa A, Komi M, Hayakawa H, Hori S \& Nishimura Y, "Pulmonary nodules at chest CT: Effect of computeraided diagnosis on radiologists detection performance", Radiology, Vol.230, No.2, (2004), pp.347-352.

[25] Jiang CL, He SW, Zhang YD, Duan HX, Huang T, Huang YC, Li GF, Ma LJ, Zhou GB \& Cao Y, "Air pollution and DNA methylation alterations in lung cancer", Oncotarget, Vol.8, (2017), pp.1369-1391.

[26] B Kassimbekova, G Tulekova, V Korvyakov (2018). Problems of development of aesthetic culture at teenagers by means of the Kazakh decorative and applied arts. Opción, Año 33. 170-186

[27] Theresa MM \& Bharathi VS, "Computer aided diagnostic (CAD) for feature extraction of lungs in chest radiograph using different transform features", Biomedical Research, (2017), pp.1-8.

[28] Shen W, Zhou M, Yang F, Yu D, Dong D, Yang C, Zang Y \& Tian J, "Multi-crop convolutional neural networks for lung nodule malignancy suspiciousness classification", Pattern Recognition, Vol.61, (2017), pp.663-673.

[29] Liu K \& Kang G, "Multiview convolutional neural networks for lung nodule classification", International Journal of Imaging Systems and Technology, Vol.27, (2017), pp.12-22.

[30] Wu L, Cheng JZ, Li S, Lei B, Wang T \& Ni DF, "Fetal ultrasound image quality assessment with deep convolutional networks", IEEE Transactions on Cybernetics, Vol.47, (2017), pp.1336-1349.

[31] Koichi S \& Hiroaki A, "Correlation between immune-related adverse events and efficacy in non small cell lung cancer treated with nivolumab", Elsevier Lung Cancer, Vol.115, (2018), pp.71-74.

[32] Shaohua C, Zhuoan C, Wenxin Q \& Liyan J, "Exosomes as a liquid biopsy for lung cancer", Elsevier, (2018), pp.46-51.

[33] Daichi F, "Efficacy and safety of nivolumab in previously treated patients with nonsmall cell lung cancer: A multicenter retrospective cohort study", Elsevier, (2018), pp.14-20. 